\title{
Avian paramyoxvirus-8 immunization reduces viral shedding after homologous APMV-8 challenge but fails to protect against Newcastle disease
}

\author{
Christian Grund ${ }^{1 *}$, Constanze Steglich², Eva Huthmann ${ }^{1}$, Martin Beer ${ }^{1}$, Thomas C Mettenleiter ${ }^{2}$
} and Angela Römer-Oberdörfer ${ }^{2}$

\begin{abstract}
Background: Protection against infection by Newcastle disease virus (NDV), also designated as avian paramyxovirus subtype-1 (APMV-1), is mediated by immune responses to the two surface glycoproteins, hemagglutinin-neuraminidase $(\mathrm{HN})$ and fusion (F) protein. Thus, a chimeric APMV-1 based vaccine that encodes APMV-8 HN- and F-proteins and expresses the hemagglutinin of avian influenza virus (AIV) H5N1, is able to protect against HPAIV H5N1 but fails to protect against NDV [PLOS One 8:e72530, 2013]. However, it is unclear whether avirulent APMV-subtypes, like APMV-8 can induce subtype-specific immunity and protect from a homologous challenge.

Findings: APMV-8 infections of 3- and 6-weeks-old specific pathogen free (SPF)-chickens did not induce any clinical signs but was associated with virus shedding for up to 6 days. Viral replication was only detected in oropharyngeal- and never in cloacal swabs. Upon reinfection with homologous APMV-8, viral shedding was restricted to day 2 and in contrast to naive SPF-chickens, only RNA but no infectious virus was recovered. No protection was induced against virulent NDV challenge, although morbidity and mortality was delayed in APMV-8 primed chickens. This lack of protection is in line with a lack of reactivity of APMV-8 specific sera to APMV-1 HN-protein: Neither by hemagglutin-inhibition (HI) test nor immunoblot analyses, cross-reactivity was detected, despite reactivity to internal proteins.

Conclusions: Immune responses mounted during asymptomatic APMV-8 infection limit secondary infection against homologues reinfection and facilitates a delay in the onset of disease in a subtype independent manner but is unable to protect against Newcastle disease, a heterologous APMV-subtype.
\end{abstract}

Keywords: APMV-8, APMV, NDV, Newcastle disease, Cross-reactivity, Cross-protection

\section{Background}

Avian paramyxoviruses (APMV) replicate within the respiratory tract and intestine of their natural avian host. They belong to the genus Avulavirus in the family Paramyxoviridae within the order Mononegavirales [1]. Currently, 12 subtypes have been identified with APMV-1 to -9 known as 'classical strains' [2] and APMV-10 to 12 recently described [3-5]. The prototypic virus, APMV-1 or Newcastle Disease virus (NDV) causes a devastating disease in poultry and represents a major threat for poultry

\footnotetext{
* Correspondence: christian.grund@fli.bund.de

${ }^{1}$ Institute of Diagnostic Virology, Friedrich-Loeffler-Institut, Federal Research Institute for Animal Health, Südufer 10, D-17493 Greifswald-Insel Riems, Germany

Full list of author information is available at the end of the article
}

production in the world. In contrast, the other APMVsubtypes are not clinically relevant for poultry and circulate largely unnoticed in wild birds [6]. Also for APMV-1, strains of low virulence are well known. They do not induce clinical signs in immune competent birds but confer protection against ND [7-9]. The generation of recombinant NDV (rNDV) containing specific alterations in the genome decreased residual virulence [10] and were also used as vector system to express genes of other pathogens, e.g. highly pathogenic avian influenza virus (HPAIV) $[11,12]$. To avoid interference by maternal NDV antibodies with vaccine vector performance, we created a chimeric virus (chNDVFHNPMV8H5) by substituting the envelope glycoproteins hemagglutinin-neuraminidase $(\mathrm{HN})$ and 
fusion protein (F) of NDV by those of APMV-8 and expressing H5 of HPAIV [13]. APMV-8 was chosen as donor of $\mathrm{HN}$ and $\mathrm{F}$ because of its apathogenicity for poultry [14], description of only weak cross-reactivity between APMV-1 and APMV-8 [15,16] and low prevalence [17-19]. Vaccination with this chimeric vector HPAIV-H5-vaccine resulted in efficient protection against HPAIV H5N1 infection in chickens with NDV specific maternal antibodies (MDA) [1]. However, evasion from maternal NDV antibodies was accompanied by a lack of protection against ND. This observation corresponds to investigations by Nayak et al. [20] describing absence of protection against NDV challenge after APMV-8 infection. The serological data of the vaccination experiments with chNDVFHNPMV8H5 as well as the APMV-8 infection suggested that APMV-8 glycoproteins were immunogenic in the host. However, it remained unclear whether an APMV-8 immune response was sufficient to induce protection against homologous APMV-8 challenge. Here we describe a set of animal experiments demonstrating, that APMV-8 infection does not prevent subsequent reinfection with homologous APMV-8 but limits viral shedding.

\section{Materials and methods}

Animal experiments

Chickens from SPF-eggs (LAH, Cuxhaven), hatched at the FLI were infected oculo-nasally at 3 weeks of age with $0.1 \mathrm{ml}$ of APMV-8/goose/Delaware/1053/76 containing $10^{6} \operatorname{TCID}_{50}(\mathrm{n}=18)$. Two days post infection (dpi) two naive chickens were introduced as sentinel birds. On each of day 2, 4, 6 and 21 dpi two inoculated chickens were sacrificed and indicated internal organs were tested for APMV-8 by RT-qPCR (primer probe sequence see Additional file 1: Table S1). Three weeks after initial infection, groups were divided, one being challenged oculo-nasally with NDV/Herts33/56 (10 ${ }^{6} \mathrm{EID}_{50} /$ animal $)$ and the other re-infected oculo-nasally with APMV-8/ goose/Delaware/1053/76 (10 $\mathrm{TCID}_{50}$ /animal). In addition, each of the two viruses at the same dose and route was

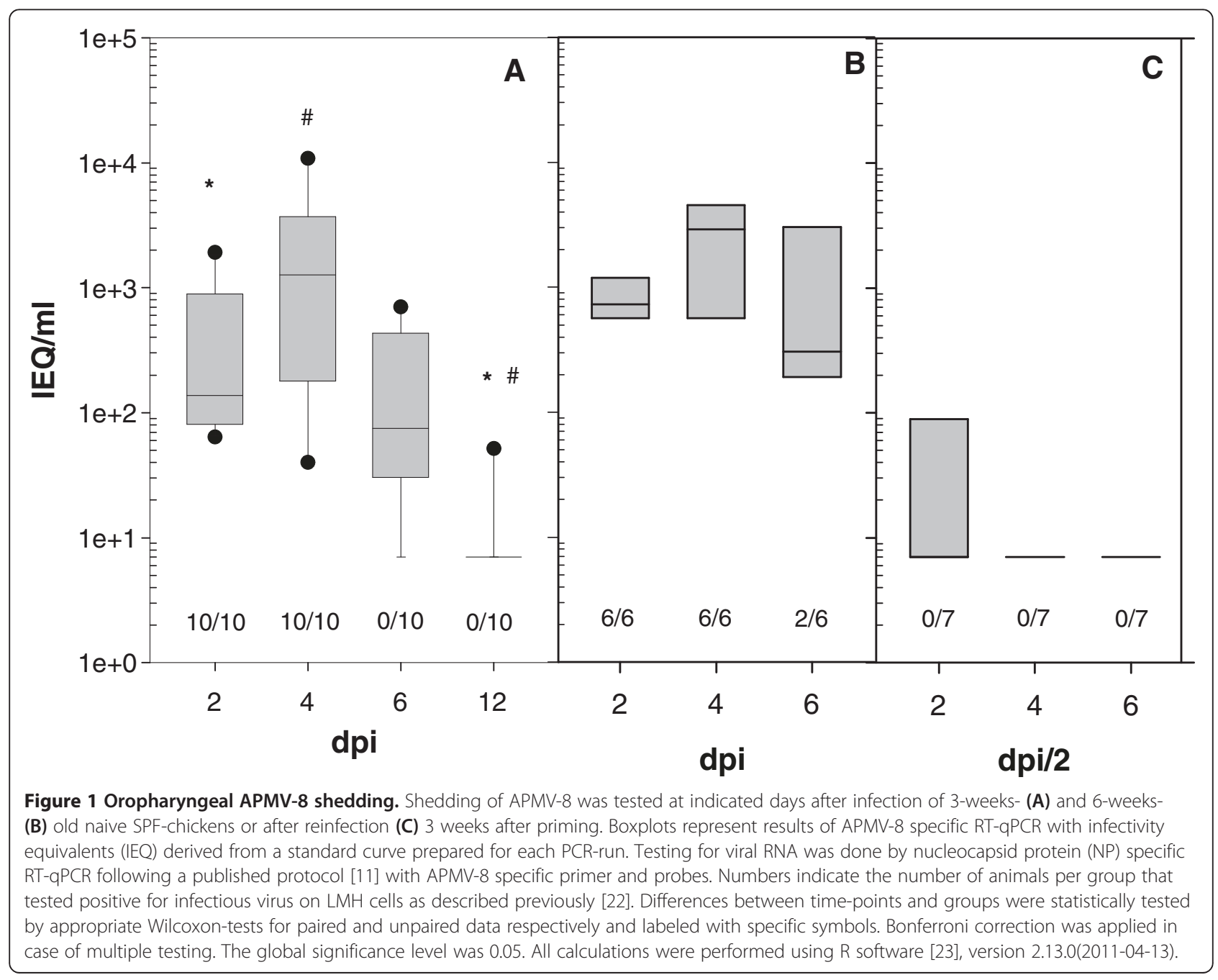


administered to 6 naive chickens from the same hatch. Animals were scored daily according their clinical condition ( 0 = healthy; 1 = sick; 2 = dead $)$ and clinical index was calculated analogous to determining intracerebral pathogenicity index (ICPI). At indicated time intervals post infection either oropharyngeal and cloacal swabs (primary infection) or combined oropharyngeal and cloacal swabs were taken for virus detection. Heparinised blood samples were obtained from all animals before vaccination, before challenge- or reinfection as well as from all surviving birds at the end of the observation period, and were tested for NDV- and APMV-8-specific antibodies using the hemagglutination inhibition (HI) assay [21]. All animal experiments were carried out in BSL3 experimental animal facilities and had been approved by the animal welfare committee (LALLF M-V/TSD/7221.3-1.1-053/10). See Additional file for specification of viruses (Additional file 2: Table S2) and APMV-subtype specific sera with their degree of cross-reactivity (Additional file 3: Table S3).

\section{Results and discussion APMV-8 infection}

Primary APMV-8 infection was conducted in 3-weeksold SPF chickens by installation of $10^{6} \mathrm{TCID}_{50}$ of APMV-8/goose/Delaware/1053/76 into the eye and nasal cavity. Virus shedding was detected in oropharyngeal swabs of all ten investigated animals on days 2 and 4 pi, positive by RT-qPCR (Figure 1) as well as virus isolation. Later, the amount of viral RNA declined and the number of chickens shedding virus decreased to 8 and 1 on day 6 and 12, respectively. In these later swab samples, virus isolation was not successful. Virus was never detected in cloacal swabs, neither by RT-qPCR nor by virus isolation. These results of confined virus replication paralleled the observation that virus RNA was not detected in lung, liver, kidney or pancreas by RT-qPCR testing two animals sacrificed on day 2, 4, 6 and 21 pi each. Viral RNA was detected in the proventriculus of one chicken on day 2 and $4 \mathrm{dpi}$ (300 and 180 genome equivalents (GEQ)/ml respectively) and in the duodenum on day 4 pi $(160 \mathrm{GEQ} / \mathrm{ml})$. Detection of a minute amounts RNA in the upper digestive tract but never in feaces points to degraded viral products rather than virus replication within these organs. These data are in agreement with observations by Kim et al. [14] detecting APMV-8 in trachea but not lung, spleen or brain of three birds sampled at day 4 after infection. Like in the former experiments, all APMV-8 infected chickens remained healthy over the entire observation period and developed like uninfected hatch mates. However, virus replication was sufficient to transmit APMV-8 to both sentinel chickens: Testing serum $21 \mathrm{dpi}$, both sentinels had seroconverted.

All APMV-8-inoculated animals seroconverted within the first week after infection and antibody titers rose to

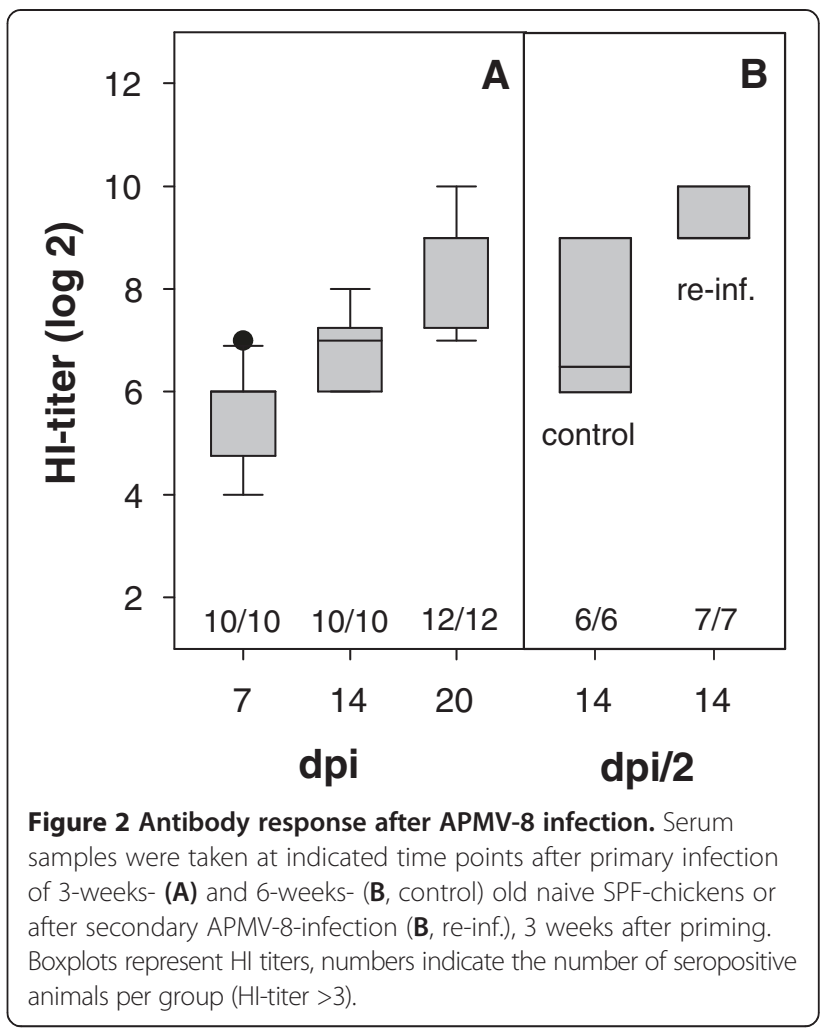

up to 8 (arithmetic mean $\varnothing 6.9 \pm 0.7)$ and $10(\varnothing 8.6 \pm 1.0)$ $\log 2$ two and three weeks post infection, respectively (Figure 2). Cross-reactivity of APMV-8 specific immune sera to APMV-1 HN was neither observed by HI (data not shown) nor immunoblot analyses (Figure 3).

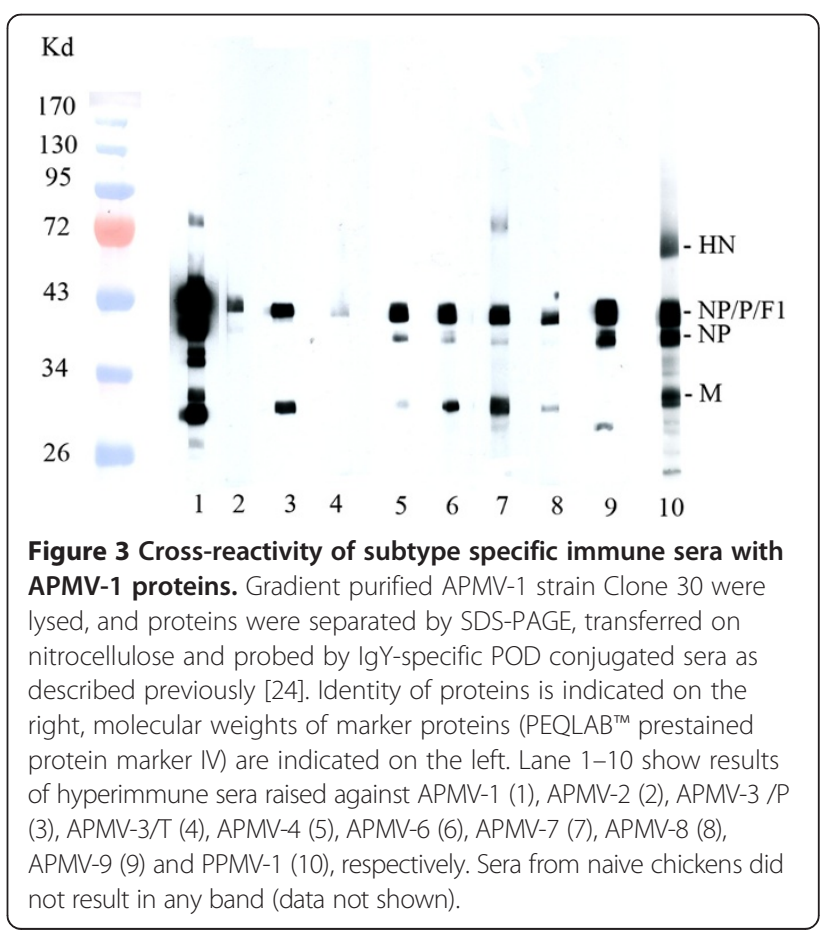


However, immunoblot analyses revealed cross-reactivity predominantly directed against internal virion proteins (Figure 3; identification of proteins see Additional file 4: Figure S1). Beside reactivity to the matrix $(\mathrm{M})$ protein, a protein band of about $50-55 \mathrm{kD}$ was reproducibly detected. Due to only minor differences in molecular weight amongst NP, P and the F1 subunit of the F protein, further differentiation of antibody specificity was not possible.

Three weeks after the initial experiment, five APMV-8 infected chickens and one sentinel animal, now six weeks old, were reinfected with $10^{6} \mathrm{TCID}_{50}$ of homologous APMV-8 strain. In addition, a group of six chickens from the same hatch but kept separately, were APMV-8 infected. As anticipated, birds inoculated with APMV-8 did not develop clinical signs but virus shedding was detected in naive 6 -weeks-old chickens from day 2 pi by RT-qPCR as well as by virus isolation comparable to shedding observed before in the 3-weeks-old animals. In contrast, in animals with previous exposure to APMV-8, virus was only detected in 3 out of 6 (47, 89 and 12087 infectivity equivalents $/ \mathrm{ml}$ ) inoculated chickens on day 2 pi and only by RT-qPCR (Figure 3). The chickens that served as sentinel for the first infection remained virus negative in all tested samples. After APMV-8 reinfection, a slight increase in antibody titer was observed, supporting the notion of limited virus-replication. It is interesting to note, that age of the animal has apparently little effect on virus replication, since virus shedding (Figure $3, \mathrm{~A}$ and $\mathrm{B}$ ) and antibody response (Figure 1, A and B control) in 3- and 6weeks-old chickens were comparable.

\section{Heterologous NDV-challenge}

APMV-8 preinfected $(n=6)$ and naive chickens $(n=6)$ were challenged with NDV in parallel. All chickens suffered severe clinical disease and swab samples tested positive for virus on day 2 pch by APMV- 1 specific RTqPCR [10] as well as by virus isolation (Figure 4). All naive SPF-chickens became sick on day 2 and were dead by day 3 . In animals that had previously been infected by APMV-8, the onset of disease was significantly delayed $(p=0.01515)$ with only one sick animal on day 2 pch and death of all chickens by day 5 . Thus, clinical index of the group with APMV-8 preinfection was lower (1.36) compared to naive chickens (1.52) after NDVchallenge (Figure 4). These data support the conclusion that cross-reactivity between internal proteins has little effect on the clinical outcome after a heterologous NDV challenge, but is able to delay the clincal course. This is in agreement with earlier experiments of Nayak and colleagues [20]: They observed mortality up to day 11 in APMV-8 preinfected chickens, compared to 9 days in non-immunized control chickens, and one out of five chickens survived challenge with 200 chicken lethal dose $_{50}\left(\mathrm{CLD}_{50}\right)$ of virulent NDV strain Texas-GB. It is interesting to note, that also after vaccination with chimeric

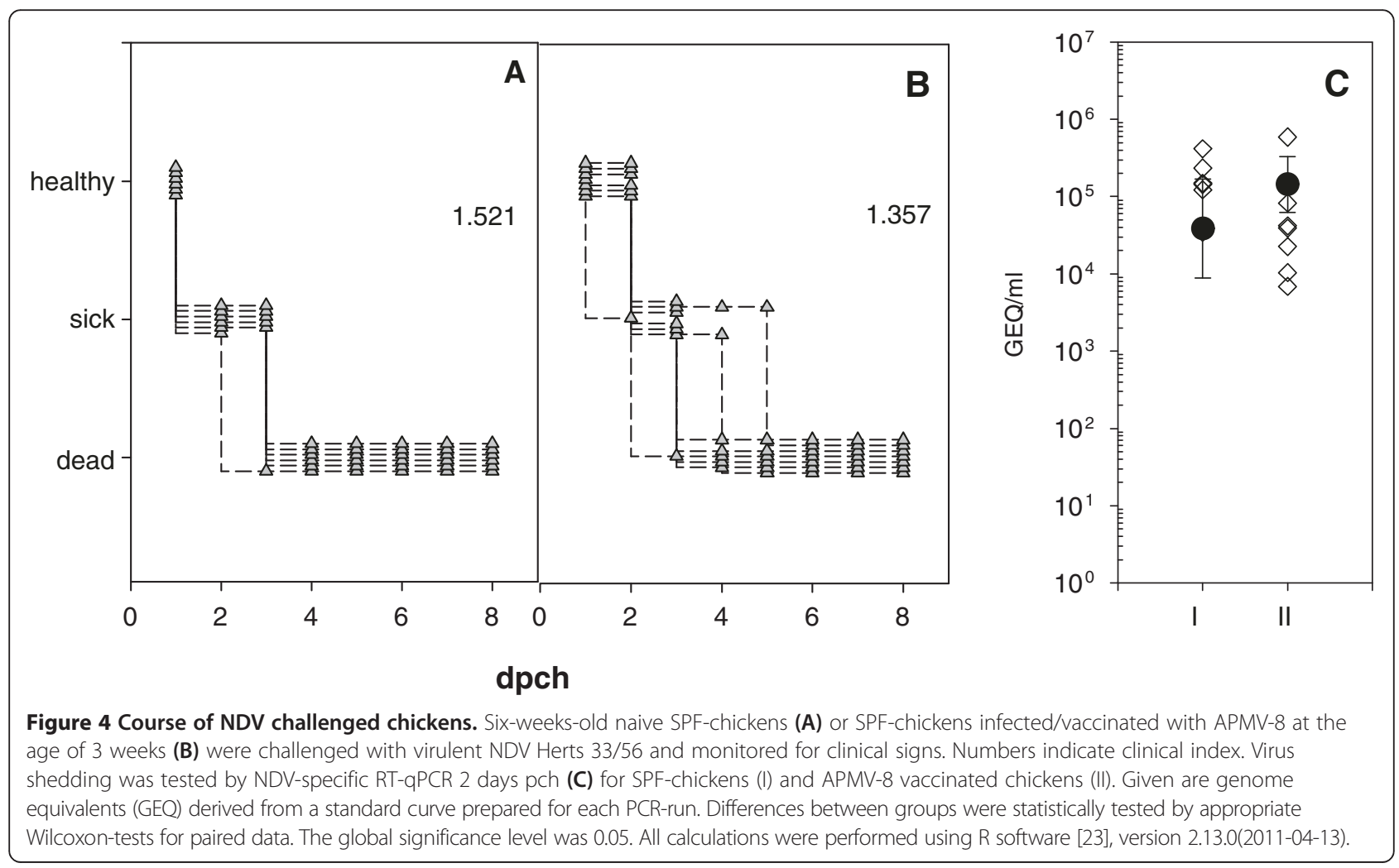


APMV-1/APMV-8 vector vaccine (chNDVFHNPMV8H5) a comparable delay of 2 days in the clinical course of ND was observed applying the same NDV challenge model [13]. Apparently, this effect of retarded clinical onset of disease is independent of the subtype. Whether this effect is associated to cross-reactivity to internal proteins needs further elucidation.

\section{Conclusions}

Active local replication of APMV-8 induced an immune response efficacious to limit APMV-8 reinfection but unable to protect against heterologous APMV-1 subtype.

\section{Additional files}

Additional file 1: Table S1. Primers and probe for APMV-8 NP-specific RT-qPCR.

Additional file 2: Table S2. Specification and source of viruses. 1 APMV-8 strain at the FLI was obtained in 1992 and working stocks for sequencing and animal experiments were derived form the 5th and 6th passage in SPF-chicken eggs. Genome was sequenced by Müller et al. (Acc. Nr. FJ 619036). Compared to published sequences by Paldurai et al. [25] (Acc. Nr. FJ 515863) 512 Single Nucleotide Polymorphism (SNP) are evident compared to 21 SNP to pintail/Wakuya/20/78; FJ215864, [25].

Additional file 3: Table S3. Cross-reactivity of APMV-subtypes tested by hemagglutination-inhibition test. Sera were produced by immunizing six-week-old SPF chickens with beta propriolactone $(0.05 \% \mathrm{v} / \mathrm{v})$ inactivated virus containing allantoic fluid emulsified with Freudschen adjuvant (Sigma). Sera were taken two weeks after the last of three immunizations and stored at $-20^{\circ} \mathrm{C}$. Results are given as $\log 2$ titer.

Additional file 4: Figure S1. Identification of specific APMV-1 proteins. Gradient purified APMV-1 strain Clone 30 were lysed, and proteins were separated by SDS-PAGE, transferred on nitrocellulose and probed by IgY-specific POD-conjugated sera as described previously [24]. Identity of proteins is indicated on the right, molecular weights of marker proteins (PEQLAB ${ }^{\mathrm{TM}}$ prestained protein marker IV) are indicated on the left. Lanes show results of chicken serum raised against PPMV-1 (1), monoclonal antibodies directed against NP- (2), P- (3); HN-protein( 4) and monospecific rabbit serum raised against F-protein (5).

\section{Competing interests}

The authors declare that they have no competing interests.

\section{Authors' contributions}

CG planned and carried out the animals experiments, analyzed test results and wrote the manuscript, CS assisted with animals experiments, performed the laboratory testing and analyses of the data, EH performed immunoblot analyses, MB helped in analyzing the data and preparation of the manuscript, TCM edited the manuscript; AR planned experiments and drafted the manuscript. All authors read and approved the final manuscript.

\section{Acknowledgments}

We like to thank Cornelia Illing for excellent technical assistance, Dr. Mario Ziller for sophisticated statistical analysis and our colleagues from the animal core facility (ATB) for diligent taking care of the animals.

\section{Author details}

${ }^{1}$ Institute of Diagnostic Virology, Friedrich-Loeffler-Institut, Federal Research Institute for Animal Health, Südufer 10, D-17493 Greifswald-Insel Riems, Germany. ${ }^{2}$ Institute of Molecular Virology and Cell Biology, Friedrich-Loeffler-Institut, Federal Research Institute for Animal Health, Südufer 10, D-17493 Greifswald-Insel Riems, Germany.

Received: 19 June 2014 Accepted: 27 September 2014 Published: 8 October 2014

\section{References}

1. Wang LF, Collins PL, Fouchier RAM, Kurath G, Lamb RA, Randall RE, Rima BK: Paramyxoviridea. In Virus Taxonomy: Ninth Report of the International Committee on Taxonomy of Viruses. Edited by King AMQ, Adams MJ, Carstens EB, Lewkowitz EJ. London: Elsevier; 2012:672-685.

2. Alexander DJ: Newcastle disease and other avian paramyxoviruses. Rev Sci Tech 2000, 19:443-462.

3. Briand FX, Henry A, Massin P, Jestin V: Complete genome sequence of a novel avian paramyxovirus. J Virol 2012, 86:7710.

4. Miller PJ, Afonso CL, Spackman E, Scott MA, Pedersen JC, Senne DA, Brown JD, Fuller CM, Uhart MM, Karesh WB, Brown $H$, Alexander DJ, Swayne DE: Evidence for a new avian paramyxovirus serotype 10 detected in rockhopper penguins from the Falkland Islands. J Virol 2010, 84:11496-11504.

5. Terregino C, Aldous EW, Heidari A, Fuller CM, De Nardi R, Manvell RJ, Beato MS, Shell WM, Monne I, Brown IH, Alexander DJ, Capua I: Antigenic and genetic analyses of isolate APMV/wigeon/Italy/3920-1/2005 indicate that it represents a new avian paramyxovirus (APMV-12). Arch Virol 2013, 158:2233-2243.

6. Alexander DJ: In Newcastle disease and other avian Paramyxoviridae infections. Diseases of poultry. 10th edition. Edited by Calnek BW. Ames: (Mosby-Wolfe iowa State University Press; 1997:541-569.

7. Goldhaft TM: Historical note on the origin of the LaSota strain of Newcastle disease virus. Avian Dis 1980, 24:297-301.

8. Hanson RP, Brandly CA: Identification of vaccine strains of Newcastle disease virus. Science 1955, 122:156-157.

9. Hitchner SB: Serendipity in science-discovery of the B-1 strain of Newcastle Disease virus. Avian Dis 1975, 19:215-223.

10. Ramp K, Topfstedt E, Wäckerlin R, Höper D, Ziller M, Mettenleiter TC, Grund C, Römer-Oberdörfer A: Pathogenicity and immunogenicity of different recombinant Newcastle disease virus clone 30 variants after in ovo vaccination. Avian Dis 2012, 56:208-217.

11. Schröer D, Veits J, Grund C, Dauber M, Keil G, Granzow H, Mettenleiter TC, Römer-Oberdörfer A: Vaccination with Newcastle disease virus vectored vaccine protects chickens against highly pathogenic $\mathrm{H} 7$ avian influenza virus. Avian Dis 2009, 53:190-197.

12. Veits J, Wiesner D, Fuchs W, Hoffmann B, Granzow H, Starick E, Mundt E, Schirrmeier H, Mebatsion T, Mettenleiter TC, Römer-Oberdörfer A: Newcastle disease virus expressing $\mathrm{H} 5$ hemagglutinin gene protects chickens against Newcastle disease and avian influenza. Proc Natl Acad Sci USA 2006, 103:8197-8202.

13. Steglich C, Grund C, Ramp K, Breithaupt A, Höper D, Keil G, Veits J, Ziller M Granzow H, Mettenleiter TC, Römer-Oberdörfer A: Chimeric Newcastle Disease Virus Protects Chickens against Avian Influenza in the Presence of Maternally Derived NDV Immunity. PLoS One 2013, 8:e72530.

14. Kim SH, Xiao S, Shive H, Collins PL, Samal SK: Replication, neurotropism, and pathogenicity of avian paramyxovirus serotypes 1-9 in chickens and ducks. PLoS One 2012, 7:e34927.

15. Cloud SS, Rosenberger JK: Characterization of nine avian paramyxoviruses. Avian Dis 1980, 24:139-152.

16. Lipkind M, Shihmanter E: Antigenic relationships between avian paramyxoviruses. I. Quantitative characteristics based on hemagglutination and neuraminidase inhibition tests. Arch Virol 1986, 89:89-111.

17. Dormitorio TV, Giambrone JJ, Guo K, Hepp GR: Detection and characterization of avian influenza and other avian paramyxoviruses from wild waterfowl in parts of the southeastern United States. Poult Sci 2009, 88:851-855.

18. Goekjian VH, Smith JT, Howell DL, Senne DA, Swayne DE, Stallknecht DE: Avian influenza viruses and avian paramyxoviruses in wintering and breeding waterfowl populations in North Carolina, USA. J Wildl Dis 2011, 47:240-245

19. Umali DV, Ito H, Katoh $H$, Ito T: Surveillance of Avian Paramyxovirus in Migratory Waterfowls in the San-in Region of Western Japan from 2006 to 2012. J Vet Med Sci 2014, 76:423-430.

20. Nayak B, Dias FM, Kumar S, Paldurai A, Collins PL, Samal SK: Avian paramyxovirus serotypes 2-9 (APMV-2-9) vary in the ability to induce protective immunity in chickens against challenge with virulent Newcastle disease virus (APMV-1). Vaccine 2012, 30:2220-2227.

21. CEC: Council Directive 92/66/EEC introducing Community measures for the control of Newcastle disease. Official Journal of the European Community L 1992, 260:1-20. 
22. Steglich C, Grund C, Röder A, Zhao N, Mettenleiter TC, Römer-Oberdörfer A: Chimeric avian paramyxovirus-based vector immunization against highly pathogenic avian influenza followed by conventional Newcastle disease vaccination eliminates lack of protection from virulent ND virus.

Trials Vaccinol 2014, 3:65-72.

23. Development Core Team: $R$ : A language and environment for statistical computing. Vienna, Austria: R Foundation for Statistical Computing; 2011. URL: http://www.r-project.org/. ISBN 3-900051-07-0.

24. Häuslaigner R, Sonnenburg J, Kothlow S, Kaspers B, Staubach C, Grund C: Evaluation of an indirect enzyme-linked immunosorbent assay to study the specific humoral immune response of Muscovy ducks (Cairina moschata) and domestic geese (Anser anser var. domestica) after vaccination against Newcastle disease virus. Avian Pathol 2009, 38:89-95.

25. Paldurai A, Subbiah M, Kumar S, Collins PL, Samal SK: Complete genome sequences of avian paramyxovirus type 8 strains goose/Delaware/1053/ 76 and pintail/Wakuya/20/78. Virus Res 2009, 142:144-153.

doi:10.1186/1743-422X-11-179

Cite this article as: Grund et al:: Avian paramyoxvirus-8 immunization reduces viral shedding after homologous APMV-8 challenge but fails to protect against Newcastle disease. Virology Journal 2014 11:179.

\section{Submit your next manuscript to BioMed Central and take full advantage of:}

- Convenient online submission

- Thorough peer review

- No space constraints or color figure charges

- Immediate publication on acceptance

- Inclusion in PubMed, CAS, Scopus and Google Scholar

- Research which is freely available for redistribution 